\title{
Phase Transformation Surfaces Analysis for SMA Around a Crack Tip with Curvature
}

\author{
Mohamed Rachid Laydi ${ }^{1}$ - Christian Lexcellent ${ }^{1}$
}

Published online: 9 September 2015

(C) ASM International 2015

\begin{abstract}
Under loading, for a shape memory alloy, the stress concentration around the crack tip, inside a plate, is a serious fracture problem. The tip shape constitutes an important data. In a recent paper (Lexcellent et al. in Int $\mathbf{J}$ Fract 169:1-13, 2011), the determination of phase transformation surfaces around a crack tip without curvature was investigated. For crack with curvature, the approximate stress field solution proposed by Creager and Paris is used (Int $\mathbf{J}$ Fract 3:247-252,1967). The knowledge of phase transformation surfaces dimensions and shapes can help us to define a fracture criterion. The study is focussed on mode I because this opening mode is the most dangerous for the structure. Also, comparisons between experiments and computing can be made. Qualitative agreement was obtained.
\end{abstract}

Keywords Mechanical behaviour - Pseudoelasticity · Twinning $\cdot$ Fracture

\section{Introduction}

Shape memory alloys (SMA) are potential materials for use in smart structures, actuators, medical devices and aeronautical materials. This is because of the very large recoverable strains (on the order of $8 \%$ for equi-atomic $\mathrm{Ni}-\mathrm{Ti}$ ) associated with their superelastic or pseudoelastic behaviour. The extended use of SMA elements which are sometimes subjected to rather complex loading, raises the issue of service life of the systems and leads us to

Christian Lexcellent

christian.lexcellent@univ-fcomte.fr

1 Département de Mécanique Appliquée, FEMTO-st, 25, rue de l'épitaphe, 25000 Besançon, France investigate SMA fracture and/or fatigue damage. As discussed by Daly et al. [1], fatigue and fracture behaviours and their possible consequences on "patients health" are of great concern in medical industry where $\mathrm{Ni}-\mathrm{Ti}$ is widely used for medicals devices like stents.

For instance, Yi and Gao [2] investigated numerically the SMA fracture toughening mechanism under mode I loading and showed that the martensitic phase transformation (MPT) increases the toughness and decreases the crack tip intensity factor. Wang et al. [3] examined the stress-induced martensite near the crack tip of a CT specimen by a FEM calculation.

Moreover, we can cite experimental work about the fracture behaviour of $\mathrm{Ni}-\mathrm{Ti}$ tubes [4]. Robertson and Ritchie measured in situ three dimensional strains, phases and crystallographic alignment ahead of a growing fatigue crack (after 100 cycles). Their measurements reveal that the majority of austenite grains were subjected to only 0.5-1.0\% elastic strain despite a macroscopic superelastic strain of 6-8\% recovery associated with the MPT.

Daly et al. [1] performed a tensile test on an edge crack specimen of austenitic $\mathrm{Ni}-\mathrm{Ti}$ using an in situ optical technique to examine the shape and the size of the phase transformation zones. They measured the fracture toughness $\mathrm{K} 1 \mathrm{c}$ at room temperature for fine-grained polycrystalline nitinol sheets.

Concerning a classical elasto-plastic material, in the frame of linear elastic fracture mechanic (LEFM) hypothesis, whatever the loading mode is chosen around the crack tip without curvature, the stress field is given in $\frac{1}{\sqrt{r}}$ (where $r$ is the distance to the crack tip) and hence singular. The material response is a plastification around this crack tip. The size of this plastic zone depending of the ductility of the material renders the LEFM hypothesis valid or not. 
About the shape memory alloys, the martensitic phase transformation replaces the elasto-plastic transition. Analytical prediction of the phase transformation onset zones was performed on a crack tip without curvature for the three modes (I, II and III) and mixed mode I+II [5]. The two cases of plane stress (CP) and plane strain (DP) were investigated. A special attention was devoted to take into account of the observed asymmetry between tension and compression for these yield surfaces modelling. It means that a simple Huber-Von Mises equivalent stress criterion is not sufficient. In fact, a correction function $f$ called $\langle\langle$ shape function $\rangle\rangle$ (multiplied by the Huber-von Mises equivalent stress) is introduced to take into account the asymmetry between tension and compression called "stress differential effect" by Raniecki and Mroz [6].

In the present investigation, a plate with a crack tip with curvature $\rho$ is studied. Creager and Paris [7] propose an approximate stress field solution which takes finite value on the crack tip. This study is linked to the possibility of experimental validation by mode I fracture tests.

\section{Problem with a Crack Tip with Curvature $\rho$ to Solve}

\section{Stress Field Around the Crack Tip}

Let us consider a plate of length $l$, width $w$, thickness $e$ and a fracture length $a$ (Fig. 1). The sample dimensions are chosen to comply with the instructions of the $\langle\langle$ stress intensity factor handbook $\rangle\rangle$ of Murakami et al. [8].

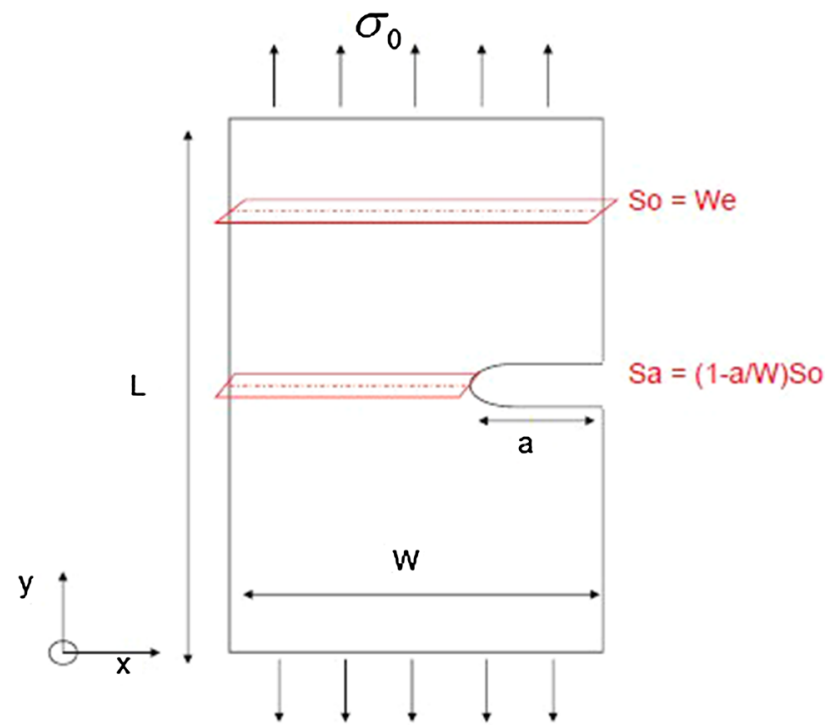

Fig. 1 Standard plate with a crack with curvature $\rho$ under mode I loading
In agreement with Irwin [9], the stress intensity factor $K_{\mathrm{I}}$, under the ratio condition $\frac{a}{w}<0.6$ can be written as

$K_{\mathrm{I}}=g\left(\frac{a}{w}\right) \sigma_{0} \sqrt{\pi a}$

with the empirical $g\left(\frac{a}{w}\right)$ expression [8]

$$
\begin{aligned}
g\left(\frac{a}{w}\right)= & 1.122-0.231\left(\frac{a}{w}\right)+10.55\left(\frac{a}{w}\right)^{2} \\
& -21.72\left(\frac{a}{w}\right)^{3}+30.39\left(\frac{a}{w}\right)^{4} .
\end{aligned}
$$

One has to note that an expression of $g$ function integrating the curvature $\rho$ in our knowledge does not exist in the literature.

Starting from the classical stress field around the crack tip for mode I (without curvature radius), Creager and Paris [7] extend the solution for finite curvature $\rho$ as

$$
\begin{aligned}
\sigma_{x x} & =\frac{K_{I}}{\sqrt{2 \pi r}}\left(\cos \frac{\theta}{2}\left(1-\sin \frac{\theta}{2} \sin \frac{3 \theta}{2}\right)-\left(\frac{\rho}{2 r}\right) \cos \frac{3 \theta}{2}\right) \\
\sigma_{y y} & =\frac{K_{I}}{\sqrt{2 \pi r}}\left(\cos \frac{\theta}{2}\left(1+\sin \frac{\theta}{2} \sin \frac{3 \theta}{2}\right)+\left(\frac{\rho}{2 r}\right) \cos \frac{3 \theta}{2}\right) \\
\sigma_{x y} & =\frac{K_{I}}{\sqrt{2 \pi r}}\left(\sin \frac{\theta}{2} \cos \frac{\theta}{2} \cos \frac{3 \theta}{2}-\left(\frac{\rho}{2 r}\right) \sin \frac{3 \theta}{2}\right)
\end{aligned}
$$

growing.

Note again that $r$ has a finite value on the crack with equation $r=\frac{\rho}{2}$ and, therefore, the stresses have a finite value, so there is no singularity.

Remember that

$$
\begin{aligned}
\sigma_{y z} & =\sigma_{z x}=0 \\
\sigma_{z z} & =0 \text { for plane stress }(C P) \\
\sigma_{z z} & =v\left(\sigma_{x x}+\sigma_{y y}\right) \text { for plane strain }(D P)
\end{aligned}
$$

Let the tensor $\boldsymbol{\sigma}$ and $\mathbf{S}_{\boldsymbol{\sigma}}$ be its deviatoric part

$$
\mathbf{S}_{\boldsymbol{\sigma}} \equiv \boldsymbol{\sigma}-\frac{\mathbf{1}}{\mathbf{3}} \operatorname{tr}(\boldsymbol{\sigma}) \mathbf{1} \text {. }
$$

In a classical way, the Huber-Von Mises equivalent stress is defined by

$\bar{\sigma} \equiv \kappa\left|\mathbf{S}_{\boldsymbol{\sigma}}\right|$, where $\kappa \equiv \sqrt{\frac{3}{2}}$ a normalisation parameter and $\left|\boldsymbol{S}_{\boldsymbol{\sigma}}\right| \equiv \sqrt{\operatorname{tr}\left(\mathbf{S}_{\sigma}{ }^{\mathrm{T}} \mathbf{S}_{\sigma}\right)}$ the tensor $\mathbf{S}_{\sigma}$ norm.

In order to take into account the asymmetrical behaviour between tension and compression in the yield phase transformation surface prediction, a parameter called $\langle\langle$ Lode parameter $\rangle\rangle$ is introduced $[10,11]$.

$$
y_{\sigma} \equiv \frac{6 \kappa}{\left|\mathbf{S}_{\sigma}\right|^{3}} \operatorname{det} \mathbf{S}_{\sigma} .
$$

Now, the problem is to give the equation of the elastic austenitic domain yield surface which is called $F(\boldsymbol{\sigma})$. In this aim, tools coming from plasticity are used. 
As the phase transformation process is independent of the pressure, one suggests to write $F(\boldsymbol{\sigma})$ as

$F(\boldsymbol{\sigma})=\bar{\sigma} f\left(y_{\sigma}\right)-\sigma_{\mathrm{c}}(T)=0$.

For a given boundary value $\sigma_{\mathrm{c}}(T)>0$, we seek to calculate the distance $r$

$r: \theta \in[0,2 \pi] \rightarrow r(\theta) \geq \frac{1}{2} \rho>0$

solution of the non linear equation (6).

\section{Huber-von Mises particular case}

One begins with the Huber-Von Mises simple case where $f=1$ in the yield surface equation (6). It means that

$\bar{\sigma}=\sigma_{c}$.

The tensor $\boldsymbol{\sigma}$ is written as function of an auxiliary variable

$z=\frac{\rho}{2 r}$.

This delivers the condition on the $z$ variable

$0 \leq z \leq 1$

and with $\frac{K_{I}}{\sqrt{2 \pi r}}=\frac{K_{i}}{\sqrt{\pi \rho}} \sqrt{z}$ under the shape

$\boldsymbol{\sigma}=\frac{K_{i}}{\sqrt{\pi \rho}} \sqrt{z} \boldsymbol{\Sigma}_{\theta}(z)$ where $\boldsymbol{\Sigma}_{\theta}(z) \equiv \mathbf{A}_{\theta}-\mathbf{z B} \mathbf{B}_{\theta}$

with

$\mathbf{A}_{\theta}=\cos \frac{\theta}{2}\left(\begin{array}{ccc}1-\sin \frac{\theta}{2} \sin \frac{3 \theta}{2} & \sin \frac{\theta}{2} \cos \frac{3 \theta}{2} & 0 \\ \sin \frac{\theta}{2} \cos \frac{3 \theta}{2} & 1+\sin \frac{\theta}{2} \sin \frac{3 \theta}{2} & 0 \\ 0 & 0 & 1-\beta\end{array}\right)$

and

$\mathbf{B}_{\theta}=\left(\begin{array}{ccc}\cos \frac{3 \theta}{2} & \sin \frac{3 \theta}{2} & 0 \\ \sin \frac{3 \theta}{2} & -\cos \frac{3 \theta}{2} & 0 \\ 0 & 0 & 0\end{array}\right)$

and also

$\beta=\left\{\begin{array}{c}1 \text { for plane stress } C P \\ 1-2 v \text { for plane strain } D P\end{array}\right\}$.

A simple calculation delivers the eigenvalues of $\mathbf{S}_{\Sigma_{\theta}}$ (deviatoric part of $\boldsymbol{\Sigma}_{\theta}(z)$ ) $\lambda_{1}=-2 \frac{\beta}{3} \cos \frac{\theta}{2}, \quad \lambda_{2}=\frac{\beta}{3} \cos \frac{\theta}{2}-\sqrt{z^{2}+\frac{1}{4} \sin ^{2} \theta}$,

$\lambda_{3}=\frac{\beta}{3} \cos \frac{\theta}{2}+\sqrt{z^{2}+\frac{1}{4} \sin ^{2} \theta}$

and the Huber-von Mises expression

$\bar{\sigma}=\frac{K_{i}}{\sqrt{\pi \rho}} \sqrt{z} \overline{\boldsymbol{\Sigma}}_{\theta}(z), \quad$ with $\overline{\boldsymbol{\Sigma}}_{\theta}(z)=3^{\frac{1}{2}} \sqrt{z^{2}+3 p_{\theta}}$

where

$p_{\theta} \equiv \frac{1}{9}\left(\beta^{2}+3 \sin ^{2} \frac{\theta}{2}\right) \cos ^{2} \frac{\theta}{2} \leq \frac{4}{27} \forall \theta$.

As a consequence, the Eq. (8) is equivalent to

$\varphi_{p}(z)=\chi \quad$ with $\chi=\frac{1}{6} \pi \rho\left(\frac{\sigma_{c}}{K_{I}}\right)^{2}$,

where $\varphi_{p}$ is defined whatever $p \geq 0$, by

$z \in R^{+} \rightarrow \varphi_{p}(z) \equiv \frac{1}{2} z\left(z^{2}+3 p\right) \in R^{+}$.

This function admits as reciprocal one $\psi_{p} \equiv \varphi_{p}^{-1}$ (obtained by the Cardan method) defined on $R^{+}$by

$\psi_{p}(\chi)=\left(\sqrt{\chi^{2}+p^{3}}+\chi\right)^{\frac{1}{3}}-\left(\sqrt{\chi^{2}+p^{3}}-\chi\right)^{\frac{1}{3}}$.

Hence by inversion, the Eq. (8) admits, whatever $\chi>0$, one solution and only one $z=\frac{\rho}{2 r}$, such that

$z=\psi_{p}(\chi)$.

However, the Eq. (7) imposes the condition

$0 \leq \psi_{p}(\chi) \leq 1$

i.e.

$\chi \leq \varphi_{p}(z=1)=\frac{1}{2}(1+3 p) \forall p \in\left[0, \frac{4}{27}\right]$.

Hence

$\chi \leq \frac{1}{2}$.

In brief, the following result is established:

Proposal 1:

Equation (8) admits a unique solution

$r=\frac{1}{2} \frac{\varrho}{\psi_{p}(\chi)}$.

Verifying condition (7) if and only if $\chi$ verifies condition (24). 


\section{General Case}

This case consists in replacing $\sigma_{c}$ by $\frac{\sigma_{c}}{f\left(y_{\sigma}\right)}$ or more simply by $\frac{\sigma_{c}}{f\left(y_{\Sigma_{\theta}}\right)}$ because

$\bar{\sigma}=\frac{K_{i}}{\sqrt{\pi \rho}} \sqrt{z} \bar{\Sigma}_{\theta}(z) \quad$ and $y_{\sigma}=y_{\Sigma_{\theta}}$

i.e.

$\bar{\sigma}=\frac{K_{i}}{\sqrt{\pi \rho}} \sqrt{z} \overline{\boldsymbol{\Sigma}}_{\theta}(z)=\frac{\sigma_{c}}{f\left(y_{\Sigma_{\theta}}\right)}$.

More precisely, one has the equivalences

$G(\boldsymbol{\sigma})=\bar{\sigma} f\left(y_{\Sigma_{\theta}}\right)=\sigma_{c} \Leftrightarrow \sqrt{z} G\left(\Sigma_{\theta}\right)=2 \kappa \chi^{\frac{1}{2}} \Leftrightarrow z=\psi_{p}\left(\chi \alpha_{\theta}(z)\right)$,

where $\alpha_{\theta}(z)$ is a continuous function of $z$ and $\theta$ defined by

$\alpha_{\theta}(z) \equiv\left|f\left(y_{\Sigma_{\theta}}\right)\right|^{-2}$.

Its explicit expression is obtained easily by combining in the expression (5) the value of $\left|\mathbf{S}_{\Sigma_{\theta}}\right|$

$\left|\mathbf{S}_{\Sigma_{\theta}}\right|=\left(\frac{2 \beta^{2}}{3} \cos ^{2} \theta+\frac{\sin ^{2} \theta}{2}+2 z^{2}\right)^{\frac{1}{2}}$

with

$\operatorname{det}\left(\mathbf{S}_{\Sigma_{\theta}}\right)=\frac{2 \beta}{3} \cos \frac{\theta}{2}\left(z^{2}+\frac{1}{4} \sin ^{2} \theta-\left|\frac{\beta}{3} \cos \frac{\theta}{2}\right|^{2}\right)$.

Moreover, one has the following framing:

$\frac{1}{8} \eta^{3} \leq \chi \alpha_{\theta}(z) \leq \frac{1}{2} \eta^{3} \quad$ where $\eta=\left(6 \chi|f(0)|^{-2}\right)^{\frac{1}{2}}$.

This result is the direct consequence of the convexity condition of the $G$ yield function (see Laydi and Lexcellent [12]) which imposes the necessary condition on the $f$ function

$3^{-\frac{1}{2}} f(0) \leq f(y) \leq 2 \times 3^{-\frac{1}{2}} f(0) \forall y \in[-1,1]$.

As reference of the single Huber-von Mises problem, the resolution of this problem consists in finding $\hat{z}$ solution of one of the following equations:

$\varphi_{p}(\hat{z})=\chi \alpha_{\theta}(\hat{z}) \Longleftrightarrow \hat{z}=\psi_{p}\left(\chi \alpha_{\theta}(\hat{z})\right)$.

Let

$\xi \equiv \psi_{\frac{4}{27}}\left(\frac{1}{8} \eta^{3}\right)$

Taking into account all the notations and hypothesis, we are able to demonstrate the following theorem.

\section{Theorem}

Let $\eta \in[0,1]$. Then, the Eq. (6) admit at least one solution $r=\frac{\rho}{2 z}$ such that

$$
\xi \leq \psi_{p}\left(\frac{1}{8} \eta^{3}\right) \leq z \leq \psi_{p}\left(\frac{1}{2} \eta^{3}\right) \leq \eta \forall \theta .
$$

Moreover, if

$\eta \leq 0.34$

then the solution is single.

\section{Proof}

Let us give a fixed value to $\theta$ and introduce the function $\widehat{w}_{\theta}(z)=w_{\theta}(z)-z \quad$ where $w_{\theta}(z)=\psi_{p}\left(\chi \alpha_{\theta}(z)\right)$,

the function $(z, q) \in R^{+} \times R^{+} \rightarrow \psi_{q}(z)$ being increasing as function of $z$ and decreasing as function of $q$. Hence, the framing (Eq. 30) and the inequality (17) give

$$
\begin{aligned}
\xi & \equiv \psi_{\frac{4}{27}}\left(\frac{1}{8} \eta^{3}\right) \leq \psi_{p}\left(\frac{1}{8} \eta^{3}\right) \leq w_{\theta}(z) \leq \psi_{p}\left(\frac{1}{2} \eta^{3}\right) \\
& \leq \psi_{0}\left(\frac{1}{2} \eta^{3}\right)=\eta .
\end{aligned}
$$

So, the auxiliary function $\widehat{w}_{\theta}(z)$ which continues in $z$, verifies the following inequalities

$0 \leq \widehat{w}_{\theta}(\xi)$ and $\widehat{w}_{\theta}(\eta) \leq 0$.

Hence, its exist at least one point $z \in[\xi, \eta]$ such that $\widehat{w}_{\theta}(z)=0$.

This gives the proof of the existence of the $\widehat{w}_{\theta}(z)$ function.

In order to show now the unicity, let us choose two separate solutions of the problem i.e. $z_{1}, z_{2} \in[\xi, \eta]$; (see the Eq. 26)

$$
\begin{aligned}
\sqrt{z_{1}} G\left(\boldsymbol{\Sigma}\left(z_{1}\right)\right) & =\sqrt{z_{2}} G\left(\boldsymbol{\Sigma}\left(z_{2}\right)\right)=2 \kappa \chi^{\frac{1}{2}} \Rightarrow\left|\sqrt{z_{2}}-\sqrt{z_{1}}\right| G\left(\boldsymbol{\Sigma}\left(z_{2}\right)\right) \\
& =\sqrt{z_{1}}\left(\left|G\left(\boldsymbol{\Sigma}\left(z_{1}\right)\right)-G\left(\boldsymbol{\Sigma}\left(z_{2}\right)\right)\right|\right)
\end{aligned} .
$$

As $G$ is positively homogeneous of order 1 (e.g. $G(\lambda \Sigma)=$ $\lambda G(\boldsymbol{\Sigma}) \forall \lambda \geq 0$, it becomes

$$
\begin{aligned}
\left|G\left(\boldsymbol{\Sigma}\left(z_{1}\right)\right)-G\left(\boldsymbol{\Sigma}\left(z_{2}\right)\right)\right| & \leq\left|G\left(\boldsymbol{\Sigma}\left(z_{1}\right)-\boldsymbol{\Sigma}\left(z_{2}\right)\right)\right| \\
& =\left|z_{2}-z_{1}\right| G\left(B_{\theta}\right) \\
& =\left|z_{2}-z_{1}\right| \sqrt{3} f(0)
\end{aligned}
$$

and

$$
\begin{aligned}
\left|z_{2}^{\frac{1}{2}}-z_{1}^{\frac{1}{2}}\right| G\left(\boldsymbol{\Sigma}\left(z_{2}\right)\right) & \leq z_{1}^{\frac{1}{2}}\left|z_{2}-z_{1}\right| \sqrt{3} f(0) \Rightarrow 2 \kappa \chi^{\frac{1}{2}}=z_{2}^{\frac{1}{2}} G\left(\boldsymbol{\Sigma}\left(z_{2}\right)\right) \\
& \leq z_{2}^{\frac{1}{2}} z_{1}^{\frac{1}{2}}\left|z_{2}^{\frac{1}{2}}+z_{1}^{\frac{1}{2}}\right| \sqrt{3} f(0)
\end{aligned}
$$


Fig. 2 Cracked plate with curvature under mode I (Application I)
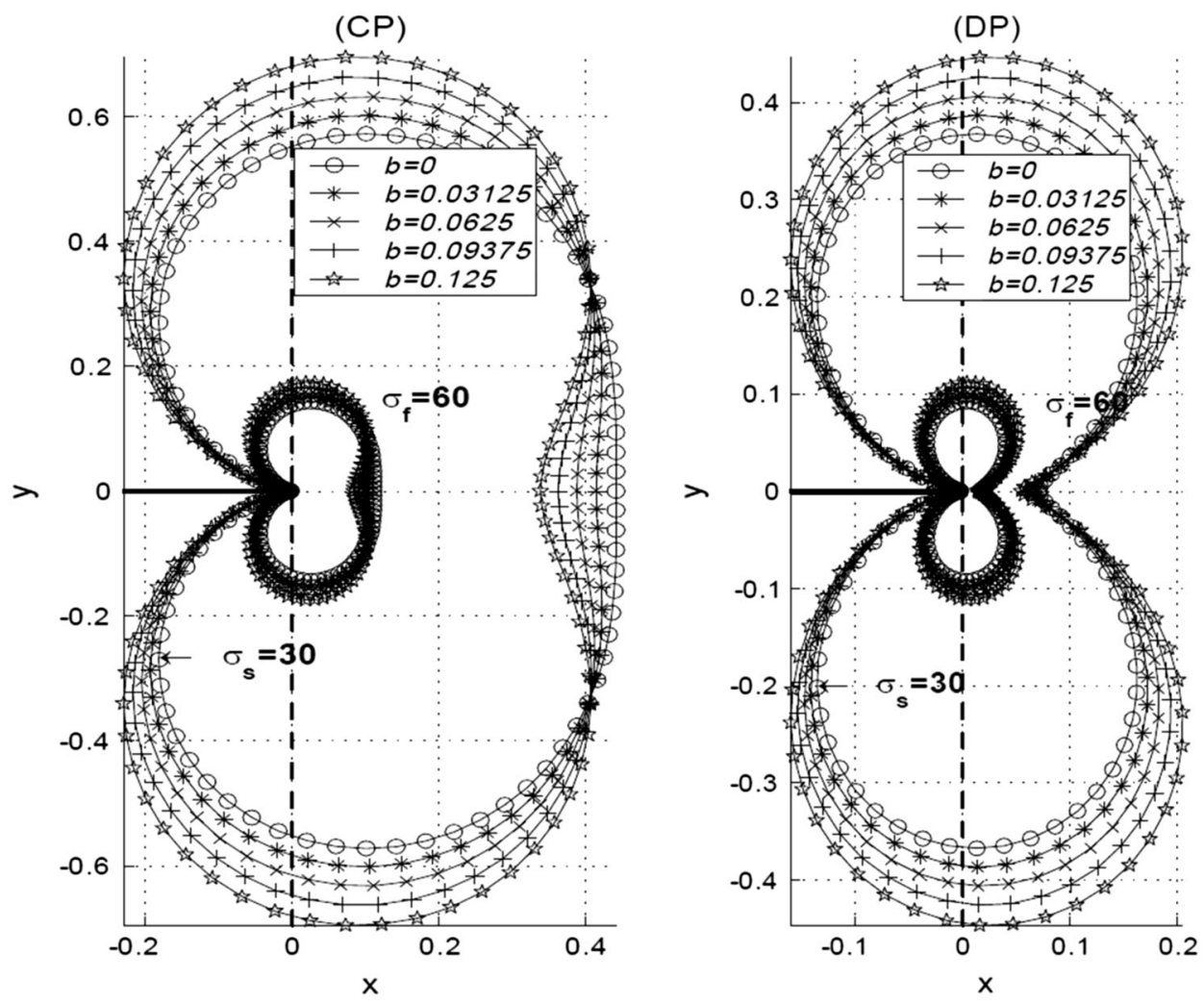

Therefore,

$\sqrt{\frac{1}{3} \eta^{3}}=\sqrt{2} \sqrt{\chi|f(0)|^{-2}} \leq z_{2}^{\frac{1}{2}} z_{1}^{\frac{1}{2}}\left|z_{2}^{\frac{1}{2}}+z_{1}^{\frac{1}{2}}\right| \leq 2 z^{\frac{3}{2}}$

for

$z=\max \left(z_{1}, z_{2}\right)$

or again from (34)

$\left(\frac{1}{12}\right)^{\frac{1}{3}} \eta \leq z \leq \psi_{p}\left(\frac{1}{2} \eta^{3}\right) \forall \theta$

So

$$
\begin{aligned}
& \frac{1}{2}\left(\frac{1}{12}\right)^{\frac{1}{3}} \eta\left(\left(\frac{1}{12}\right)^{\frac{2}{3}} \eta^{2}+3 p\right) \\
& \quad=\varphi_{p}\left(\left(\frac{1}{12}\right)^{\frac{1}{3}} \eta\right) \leq \frac{1}{2} \eta^{3} \Rightarrow \sqrt{3 p \frac{12^{\frac{2}{3}}}{11}} \leq \eta \forall \theta
\end{aligned}
$$

Let make the choice

$$
\theta=2 \operatorname{arcos} \sqrt{\frac{1}{6} \beta^{2}+\frac{1}{2}}
$$

in such way $p$ attains it maximum at the value

$p=\frac{1}{108}\left(\beta^{2}+3\right)^{2}$.
Then

$0.34<3 \sqrt{3 \times \frac{1}{108} \frac{12^{\frac{2}{3}}}{11}} \leq\left(\beta^{2}+3\right) \sqrt{3 \times \frac{1}{108} \frac{12^{\frac{2}{3}}}{11}} \leq \eta$

that is absurd.

\section{Applications}

Let the following be the material parameters:

$v=0.3, \rho=0.5(\mathrm{~mm})$,

$K_{\mathrm{I}}=50(\mathrm{MPa} \sqrt{m})$

$\sigma_{\mathrm{c}}=\left\{\begin{array}{l}\sigma_{s}=30(\mathrm{MPa}) \text { start } \\ \sigma_{f}=60(\mathrm{MPa}) \text { end }\end{array}\right.$ stress phase transformation $\}$

\section{Application I}

Let $f\left(y_{\Sigma}\right)$ be an affine function in $y_{\Sigma}$.

$f\left(y_{\Sigma}\right)=1+b y_{\Sigma}$.

Laydi and Lexcellent [13] show that the $G(\boldsymbol{\Sigma})$ function is convex in $\Sigma$ if $b \in\left[0, \frac{1}{8}\right]$. .

The verification of the condition (35) needs some elementary calculations: 


$$
\begin{aligned}
\eta & =\left(6 \chi|f(0)|^{-2}\right)^{\frac{1}{2}}=\left(\pi|f(0)|^{-2} \rho\left(\frac{\sigma_{\mathrm{c}}}{K_{\mathrm{I}}}\right)^{2}|f(0)|^{-2}\right)^{\frac{1}{2}} . \\
& =\left\{\begin{array}{c}
0.08 \text { start phase transformation } \\
0.13 \text { end phase transformation }
\end{array}\right\}<0.34
\end{aligned}
$$

In the two cases, the condition of unicity of the solution is verified. The zones of phase transformation for plane stress (CP) and plane strain (DP) conditions are shown as function of the $b$ parameter on Fig. 2 .

\section{Application II}

Let

$f\left(y_{\Sigma}\right)=\cos \left(\frac{1}{3} \arccos \left(1-a\left(1-y_{\Sigma}\right)\right)\right)$.

A new time, Laydi and Lexcellent [13] have shown that $G(\boldsymbol{\Sigma})$ is convex if $a \in[0,1]$.

The condition (35) is verified whatever $a$ is belonging to $[0,1]$. The zones of phase transformation for plane stress (CP) and plane strain (DP) conditions are shown as function of the $b$ parameter on Fig. 2. The zones of phase transformation for plane stress (CP) and plane strain (DP) conditions are shown as function of the a parameter on Fig. 3.

\section{Comparison Between Experiments and Predictions}

One has to underline that there is not important difference in the phase transformation zones exhibited for the two chosen shape functions e.g. "affine" (Fig. 2) or "arcos"
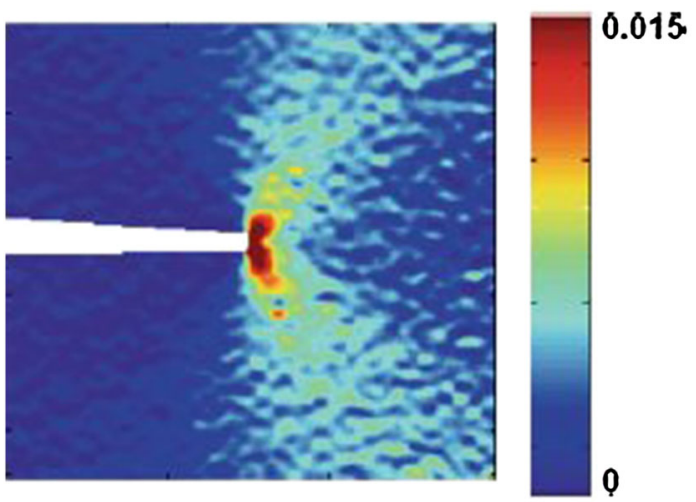

Fig. 4 Measurement of $\varepsilon_{y y}$ obtained by DIC at the crack tip for $K_{\mathrm{I}}=44 \mathrm{Mpa} \sqrt{m}[1]$
Fig. 3 Cracked plate with curvature under mode I (Application II)
(CP)

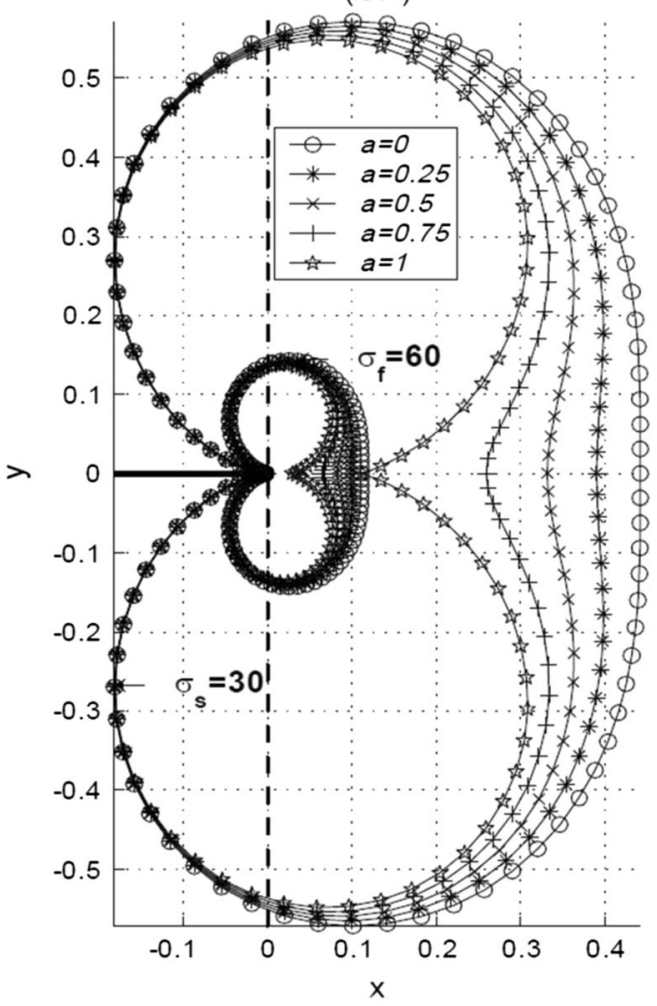

(DP)

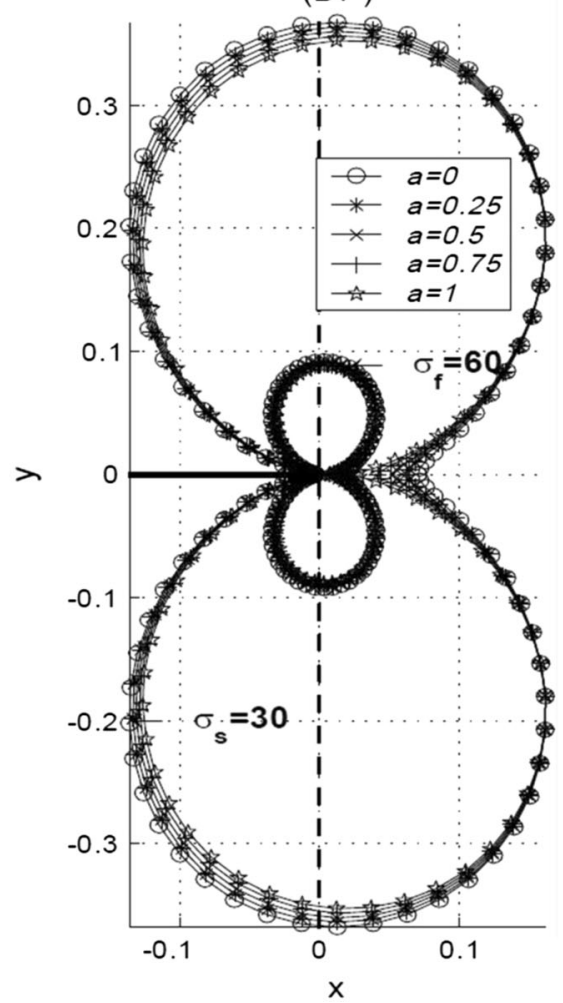


Fig. 5 Overall photo of a plate with a curvature radius under mechanical loading (FML) [14]

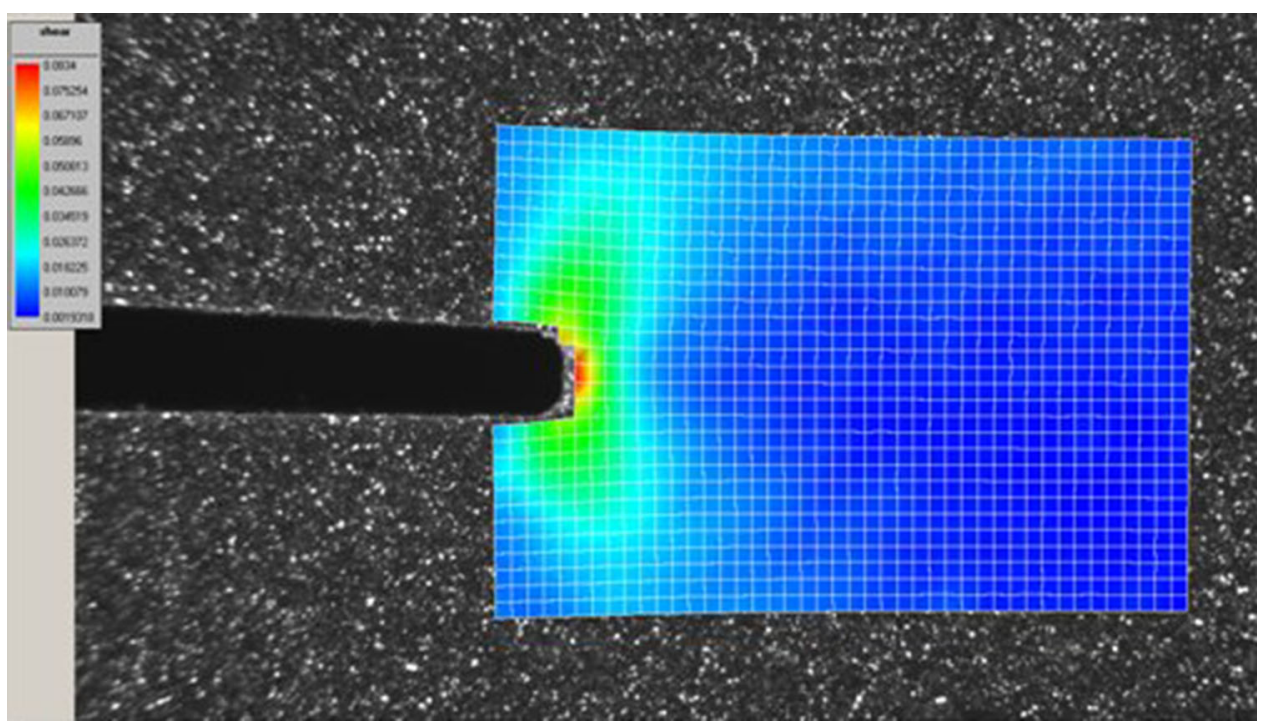

one (Fig. 3) . The impact of the parameters of asymmetry $b$ and $\mathrm{a}$ is negligible in plane strain and effective in plane stress.

Moreover, coupled thermomechanical problems can be solved by digital image correlation (DIC). Thus, Daly et al. [1] measured by DIC deformations around the crack tip on nitinol thin plates. This $\langle\langle$ in situ $\rangle\rangle$ optical method provides information about the phase transformation zones by locating deformations. The choice of the $\langle\langle$ linear elastic fracture mechanics $\rangle\rangle$ (LEFM) hypothesis seems justified by the small size of the transformation zones as shown on Fig. 4.

The shape of the lobes of the transformation surface inclined at around $60^{\circ}$, validates taking into account the asymmetry between tension and compression in the calculations.

Tensile tests have been performed in the SYMME laboratory of Annecy on samples exhibiting different radii of curvature [14].

Depending on the dimensions of the sample and on the curvature radius, we can calculate the stress intensity factors by making use of correction coefficient from Eq. (2).

$\begin{array}{ccccc}\rho(\mathrm{mm}) & 0.25 & 0.5 & 1 & 1.5 \\ K_{i \mathrm{c}}(\mathrm{MPa} \sqrt{m}) & 91 & 102 & 108 & 115\end{array}$

Its means that the intensity factor $K_{\mathrm{Ic}}$ is slighty dependant of the curvature radius $\rho$.

The shape of the transformation zones shown on the Fig. 5 for plane stress conditions is consistent with our predictions.

\section{Conclusion}

Comparison between experiments and predictions for mode I shows us a qualitative agreement.
Moreover, crack tip stress-induced martensitic transformation and the resulting stress distribution in a Ni-Ti have been also analysed by Maletta et al. [15]. Their recent analytical model on SMA pseudoelasticity occurring in the crack tip region "causes a complex and unsual stress distribution" [16]. It means that the problem to solve is complex. The transition of phase transformation mechanisms at microscale to macroscale is not entirely understood. Now with stress field around the crack tip, given a new time by Creager and Paris [7], the mode II is investigated. The calculations seem a little more complicated.

Investigations with mixed mode (I+II) loading conditions will end this next work.

\section{References}

1. Daly S, Miller G, Ravichandran G, Bhattacharya K (2007) An experimental investigation of crack initiation in thin sheets. Acta Mater 55:6322-63330

2. Yi S, Gao S (2000) Fracture toughening mechanism of shape memory alloys due to martensitic transformation. Int $\mathrm{J}$ Solids Struct 37(38):5315-5327

3. Wang XM, Wang YF, Baruj A, Eggeler G, Yue ZF (2005) On the formation of martensite in front of cracks in pseudoelastic shape memory alloys. Mater Sci Eng A 394(1-2):393-398

4. Robertson SW, Ritchie RO (2007) In vitro fatigue-crack growth and fracture toughness behavior of thin-walled superelastic nitinol tube for endovascular stents: a basis for defining the effect of crack-like defects. Biomaterials 28:700-709

5. Lexcellent C, Laydi RM, Taillebot V (2011) Analytical prediction of the phase transformation onset zone at a crack tip of a shape memory alloy exhibiting asymmetry between tension and compression. Int J Fract 169:1-13

6. Raniecki B, Mroz Z (2008) Yield or martensitic phase transformation conditions and dissipation functions for isotropic, pressure-insensitive alloys exhibiting sd effect. Acta Mech 195:81-102 
7. Creager M, Paris PC (1967) Elastic field equations for blunt cracks with reference to stress corrosion cracking. Int $\mathbf{J}$ Fract 3:247-252

8. Murakami Y (1987) Stress intensity factor handbook. The society of material science. Pergamon Press, Oxford

9. Irwin GR (1957) Analysis of stress and strains near the end of a crack traversing a plate. J Appl Mech 24:361-364

10. Orgeas L, Favier D (1998) Stress-induced martensitic transformation of a niti alloy in isothermal shear, tension and compression. Acta Mater 46(15):5579-5591

11. Vacher P, Lexcellent C (1991) Study of pseudo-elastic behavior of polycristalline shape memory alloys by resistivity measurements and acoustic emission. In: Proceedings of ICM VI, Kyoto, Japan
12. Laydi MR, Lexcellent C (2012) Rice local phase angle study for a delamination problem between a shape memory alloy and an elastic material. Arch Ration Mech Anal 204(3):977-1007

13. Laydi MR, Lexcellent C (2010) Yield criteria for shape memory materials: convexity conditions and surfaces transport. Math Mech Solids 15(2):165-208

14. Taillebot V (2012) Contribution à l'étude de la rupture desalliages à mémoire de forme. $\mathrm{PhD}$ thesis, Universite de Franche Comte

15. Maletta C, Sgambitterra E, Furgiuele F (2013) Crack tip stress distribution and stress intensity factor in shape memory alloys. Fatigue Fract Eng Mater Struct 36:903-912

16. Maletta C, Furgiuele F (2011) Fracture control parameters for niti based shape memory alloys. Int J Solids Struct 48:1658-1664 